\title{
Perioperative bleeding in patients undergoing liver transplantation
}

\author{
Małgorzata H. Starczewska, Dorota Giercuszkiewicz, Grzegorz Niewiński, Andrzej Kański \\ IIDepartment of Anaesthesia and Intensive Care, Central Teaching Hospital, \\ Medical University of Warsaw, Poland
}

\begin{abstract}
Liver transplantation (LT) remains one of the most challenging surgical procedures. For many years uncontrolled bleeding and catastrophic haemorrhages were one of the major causes of perioperative mortality and morbidity. During the past fifty years, significant progress in surgical technique and perioperative management has led to a marked change in transfusion practice over time, where up to $79.6 \%$ of LTs in experienced transplant centers are performed without any blood product transfusion. Despite this, perioperative bleeding and transfusion requirements remain potent predictors of patient's mortality, as well as postoperative complications and graft survival. The major impact of blood product transfusion on LT recipients outcomes implies that all patients on waiting lists should be carefully screened for the presence of risk factors of perioperative bleeding. Although multiple predictors of transfusion requirements during recipients have been identified, no predictive model validated across centers has been constructed. The most suitable strategies to reduce intraoperative blood loss in this group should be employed on a case-to-case basis. This paper aims to summarize the most up-to-date evidence in the management of haemostasis in LT recipients.
\end{abstract}

Key words: liver transplantation, perioperative management, haemostasis, bleeding

Anaesthesiology Intensive Therapy 2016, vol. 48, no 1, 34-40

Liver transplantation (LT) remains one of the most challenging surgical technique. For many years, uncontrolled bleeding and catastrophic haemorrhages were one of the major causes of perioperative mortality and morbidity. During more than the fifty years of experience gained since the first LT was performed by Thomas Starzl in 1963, an improvement in surgical techniques and anaesthetic management has led to a marked change in transfusion practices over time. A recently published retrospective analysis of data from the Mayo Clinic in Rochester has shown that over the last two decades there has been a significant decrease in the amount of perioperatively transfused red blood cells (RBCs), fresh frozen plasma (FFP), platelets, cryoprecipitate and intraoperative autotransfusions [1]. Interestingly, this was noted despite the fact that transfusion triggers remained the same over time [1]. Massicotte et al. [2] reported that currently up to $79.6 \%$ of LTs are performed without any blood product transfusion. The major impact of blood product transfusion on LT recipient outcomes implies that all patients on waiting lists should be carefully screened for the presence of risk factors of perioperative bleeding. The most suitable strategies to reduce intraoperative blood loss in this group should be employed on a case-to-case basis. This paper aims to summarize the most up-to-date evidence in management of haemostasis in LT recipients.

\section{HAEMOSTATIC ALTERATIONS IN LIVER TRANSPLANT RECIPIENTS}

The change in transfusion practices was possible due to a better understanding of alterations in the haemostatic system in LT recipients. The liver is a key organ in maintaining haemostatic balance in the human body. Historically, patients with chronic and acute liver failure were believed to have increased risk of spontaneous and procedure-related bleeding. Modern viscoelastic tests have proved that, in fact, more than $15 \%$ of LT recipients are hypercoagulable at the beginning of the procedure [3]. The liver synthesizes all numbered clotting factors, including fibrinogen with the exception of factor VIII and factor XIII [4]. It also produces major anticoagulant proteins - protein $\mathrm{C}$, protein $\mathrm{S}$ and 
antithrombin, as well as components of the fibrinolytic system like plasminogen and alpha-2-antiplasmin [4]. The liver is also the main site of thrombopoietin synthesis (a major growth factor regulating platelet production) and contributes to erythropoietin secretion [5]. Typically, in patients with advanced liver disease concentration of almost all procoagulant factors, except for von Willebrand factor (WF) and factor VIII, markedly decreases [6]. This is associated with a fall in an endogenous anticoagulant factors like protein $C$, protein $\mathrm{S}$ and antithrombin, along with increased concentrations of nitric oxide and prostacyclin [6]. Thrombocytopaenia occurs frequently, mainly due to reduced thrombopoietin concentration, as well as splenic sequestration of platelets in patients with hypersplenism [7]. Bleeding tendency due to low platelets might be at least partially counterbalanced by a significant increase in VWF levels, due to its increased synthesis in the endothelium [6]. Moreover, levels of VWF cleaving protease - ADAMTS-13 are significantly reduced, contributing to further rise in VWF [6]. Although in stable cirrhosis, levels of tissue plasminogen activator (t-PA) and plasminogen activator inhibitor (PAI-1) achieve a new balance, during the anhepatic phase of LT there is a tendency towards increased fibrinolysis due to reduced clearance of t-PA with relatively stable concentrations of PAI-1 [6]. This might be further exacerbated after reperfusion due to enhanced t-PA release from the graft endothelium [8]. In patients with acute liver failure inhibition of fibrinolysis becomes a marked feature due to a rise in PAI-1 levels [9]. Therefore, as both procoagulant and anticoagulant pathways are affected in patients with advanced liver disease, currently, the concept of "rebalanced haemostasis" is more widely applied. This implies that coagulation management should be based on individual patient haemostatic system assessment [10].

\section{BLOOD TRANSFUSIONS AND PATIENT OUTCOMES}

Changes in transfusion practices during LT have markedly affected patients' outcomes. Blood transfusion has proved to be a significant risk factor for increased mortality after LT $[2,11]$. Intraoperative transfusion of at least 6 units of RBCs decreases survival rates during medium and longterm follow-up [12]. A similar pattern was observed in the transfusion of any amount of FFP and platelets, irrespectively of the amount of transfused RBCs $[13,14]$. The exact mechanism linking increased mortality with blood transfusion is most likely multifactorial. High intraoperative transfusion requirements might be a surrogate of the severity of the liver disease and an indicator of technical difficulties during surgery, both of which will affect outcomes. Other theories suggest the role of circulatory overload, immune mechanisms, transfusion-associated reaction or transmission of infection [8]. Pereboom et al. demonstrated that platelet transfusion is associated with increased postoperative mortality due to a higher prevalence of acute lung injury (ALI) [15]. In another paper, Benson et al. showed that only high-plasma-containing blood products (FFP and platelets) were associated with an increase risk of transfusion-related ALI [16]. RBCs transfusion has been independently correlated with the rate of postoperative infections in the unitdependent manner [16]. Both transfusion-related ALI and postoperative nosocomial infection were associated with markedly increased hospital length-of-stay, as well as inhospital mortality [16]. The number of transfused RBCs units during LT is also a predictor of early surgical re-intervention, which in turn increases postoperative mortality three-fold [17]. RBCs transfusion is also negatively correlated with graft survival during the 1 and 5 year follow-ups [14].

\section{PREDICTORS OF TRANSFUSION REQUIREMENTS}

Although multiple predictors of transfusion requirements during LT have been identified, no predictive model validated across centers has been constructed. Risk factors of perioperative bleeding can be divided into recipient, surgery and graft-related.

\section{RECIPIENT-RELATED PREDICTORS OF BLOOD TRANSFUSION}

Amongst the general patient factors, the recipient's age and body surface area were identified as an independent predictors of the amount of RBCs transfused, the latter being proved in patients receiving a living donor transplant [18, 19]. In addition, the impact of the severity of the disease assessed with the Child-Pugh score and Model of End-Stage Liver Disease (MELD) score was frequently studied, yielding inconsistent results. Although Mangus et al. [20] have suggested that the MELD score is an independent predictor of intraoperative blood loss, as well as the number of RBCs transfused, this has not been confirmed in subsequent studies [21]. Interestingly, Roullet et al. have demonstrated that the Child-Pugh class, but not MELD score is an independent predictor of transfusion requirements [22]. This has further been validated in subsequent studies [23].

Preoperative haemoglobin level remains one of the most consistent independent variables affecting intraoperative blood transfusion during LT [21-25]. In addition, platelet value has been found to be associated with a bleeding risk [25]. Complex alterations of haemostasis in LT recipients makes classic diagnostic tests like the prothromin time (PT)/ /international normalized ratio (INR) and activated partial thromboplastin time (aPTT) of little use, as they poorly correlate with the bleeding risk during LT [9, 10, 21-24]. A single study has found that initial fibrinogen levels were associated with intraoperative blood loss and the amount of transfused RBCs [20]. In addition, the concentration of 
fibrin degradation products were independent predictors of high blood loss during LT [26].

\section{SURGERY-RELATED PREDICTORS OF BLOOD TRANSFUSION}

Surgical techniques were considered an important predictor of perioperative blood loss. The classic technique utilizing a venovenous bypass was initially suggested to be superior due to better haemodynamic stability, as well as reduced blood loss [27]. However, this has not been confirmed in further studies, which yield contrary results [28]. Furthermore, a novel piggyback technique was demonstrated to lower transfusion requirements in comparison to the conventional technique, which was explained by a less extensive dissection area, the avoidance of artificial bypass circuit, as well as a shorter warm ischaemia time affecting graft function [29]. On the other hand, a recently published Cochrane review did not confirm the superiority of one technique over the other [30]. Other surgical factors related with increased blood transfusion during $L T$ include a history of previous upper abdominal surgery, portal vein thrombosis and, in children, previous portoenterostomy $[26,28]$. Although the duration of surgery was identified as independent predictor of intraoperative blood loss and the amount of RBCs transfused, the exact direction of this relationship has not been established as longer surgery time may also be a indicator of increased intraoperative difficulties, which also could be a consequence of increased bleeding [20]. Moreover, the level of experience of the transplant team has also been claimed to impact the risk of perioperative bleeding but this has not been widely validated [28].

\section{GRAFT-RELATED PREDICTORS OF BLOOD TRANSFUSION}

Amongst graft-related variables, the donor's older age is associated with a higher risk of massive transfusion [12]. The use of living donor grafts is also considered an independent predictor of RBCs transfusion, an aspect which has been well demonstrated in the paediatric population [31]. The likely explanation of this relationship is thought to be connected to the raw edges of partial liver grafts leaving them prone to surface bleeding. Other technical factors, such as the decreased size of the donor liver, portal vein hypoplasia and an inadequate graft-recipient body weight ratio were associated with transfusion requirements in several studies $[28,32]$. Prolonged cold ischaemia time and poor graft function due to decreased production of coagulation factors also significantly increase the risk of massive transfusion [28].

Due to great inter-center variability and different endpoints utilized in studies assessing risk factors of perioperative blood loss and predictors of transfusion requirements during $L T$, it is suggested that each transplant center should aim to identify a center-specific stratification scale [26].

\section{MANAGEMENT OF PERIOPERATIVE BLEEDING}

Given the above-mentioned impact of perioperative bleeding on the morbidity and mortality of LT recipients, methods to decrease perioperative blood loss and transfusion requirements in this population have been extensively studied over the last 30 years. Unfortunately, the vast majority of the evidence comes from single center studies, with significant heterogeneity amongst the analyzed populations, as well as a variety of transfusion protocols and practices. Therefore, it yields rather low quality evidence, even if the data are pooled for meta-analyses [33]. Amongst specific actions to minimize perioperative blood loss and transfusion requirements during $\mathrm{LT}$, we can distinguish nonpharmacological and pharmacological interventions.

\section{NON-PHARMACOLOGICAL INTERVENTIONS}

The management of patients undergoing LT poses a great challenge to the anaesthetist. During the intraoperative phase, the standard measures to decrease blood loss, such as avoiding hypothermia and acidosis, are widely recommended [34]. Fluid management is considered a key player in haemostatic management during liver surgeries. Avoiding excessive fluid transfusions and maintaining low central venous pressure (CVP) during partial hepatectomy is a well-established measure to minimize intraoperative blood loss [35]. Fluid restriction not only helps one to maintain low CVP but also prevents dilutional coagulopathy associated with excessive transfusion of crystalloids and colloids. In LT recipients, it has been demonstrated that haemodilution with either normal saline or hydroxyethyl starch (HES) leads to a significant reduction in maximum clot firmness (MCF), an effect even more pronounced in patients receiving HES [36]. Therefore, restrictive fluid transfusion protocols can help one to reduce intraoperative blood loss and transfusion requirements. It is also worth mentioning that since 2014, according to the European Medicines Agency, the use of HES is contraindicated in patients with liver failure.

Although the beneficial effect of low CVP on perioperative bleeding is explained by lowering portal pressure, relative hypovolaemia might also increase the risk of significant tissue hypoperfusion, air embolism, as well as acute renal failure. Massicotte et al. [37] demonstrated that the use of phlebotomy to reduce CVP without normovolaemic haemodilution during the pre-anhepatic phase was associated with significant reduction in blood loss and an increase in the percentage of patients managed without intraoperative transfusions. This was associated with only a transient rise in serum creatinine levels 72 hours postoperatively, in comparison to the preoperative values, which was further 
normalized 5 days after LT [37]. Another way of reducing CVP and portal pressure is through the cautious use of diuretics. Due to its osmotic activity, mannitol is believed to be particularly beneficial in preventing liver congestion after reperfusion. Although in many centers (including ours) a bolus dose of mannitol $\left(0.5-1 \mathrm{~g} \mathrm{~kg}^{-1}\right)$ is administered during the pre-anhepatic phase routinely, unless contraindicated, its impact on blood transfusion requirements has not been evaluated in prospective studies. Other strategies to maintain low CVP and avoid portal congestion include the use of low tidal volumes $\left(6-8 \mathrm{~mL} \mathrm{~kg}^{-1}\right)$ and avoiding high positive end-expiratory pressure [38]. There is less evidence regarding optimal CVP values during the post-anhepatic phase. While maintaining adequate organ perfusion is crucial for graft functioning, high venous pressures can lead to graft congestion and poor graft function. This issue was addressed in a study conducted by Cywinski et al. [39]. They found no impact of CVP (CVP $<10 \mathrm{~mm} \mathrm{Hg} \mathrm{vs} \mathrm{CVP} \geq 10 \mathrm{~mm} \mathrm{Hg})$ during the post-anhepatic phase on the estimated blood loss and the amount of blood products transfusions. Moreover, it did not affect graft function, graft survival and perioperative mortality. In the era of modern haemodynamic monitoring devices, more complex protocols targeting not only preload, but also cardiac output or tissue oxygen delivery might become an interesting strategy to reduce perioperative blood loss. Data from prospective studies in this population are still expected.

It has been discussed above that haemostatic alterations in patients undergoing LT are complex and that standard coagulation tests do not reflect the functional haemostatic status. Therefore, the use of modern viscoelastic tests such as thromboelastography (TEG) or rotational thromboelastometry (ROTEM), have gained increasing interest in this population of patients. They allow to assess humoral and cellular components of the haemostatic system (both coagulation and fibrinolysis), helping to identify the cause of intraoperative bleeding, targeting specific problems and evaluating management effectiveness. A retrospective analysis of single center data revealed that in patients with a foreseeable risk of bleeding or those with high intraoperative blood loss, the intraoperative use of thromboelastometry was associated with a significant reduction in the amount of intraoperative blood product transfusions, as well as a reduction in perioperative complications and better graft function [40]. The first prospective trial comparing TEGguided with clinician-directed transfusion protocols in LT recipients have revealed that TEG use was associated with a significantly lower use of FFP and a trend towards less blood loss, with no differences in two-year patient survival [41]. Additionally, in another trial, ROTEM was found beneficial in guiding fibrinogen concentrate transfusions, thus leading to a significant decrease in RBCs, FFP and platelet concentrate transfusions [42]. Therefore, in the recent guidelines of the European Society of Anaesthesthesiology it has been recommended to use perioperative global coagulation tests (TEG/ROTEM) for targeted management of coagulopathy in patients undergoing LT [34].

The impact of the surgical team's experience level and surgical techniques utilized during LT was discussed above. Another important surgical measure to reduce perioperative blood product transfusions is use of intraoperative cell salvage (CS). In a prospective survey conducted by Massicotte et al. [43] in patients undergoing LT, the use of CS was associated with saving two RBCs unit transfusions per patient and thus also reducing costs. Nowadays, however, as an increasing amount of transfusion-free LTs are performed, a "stand by" set up rather than routine application of CS is recommended [34]. It also must be emphasized that as salvaged washed erythrocytes do not contain clotting factors or platelets, haemostatic replacement therapy must be managed accordingly. Few case reports and case series have suggested that the use of CS might increase the risk of acute respiratory distress syndrome, acute renal failure or disseminated intravascular coagulopathy due to debris released from salvaged erythrocytes [8]. This has not been confirmed in a subsequent study [44]. Another potential risk associated with CS is the bacterial contamination of blood suctioned from the surgical field. The above-mentioned study showed that despite the fact that blood washing was not able to eliminate all bacterial contamination, autotransfusion of salvaged blood, was not associated with an increased rate of positive blood cultures and risk of postoperative infections [44]. To minimize the risk of bacterial contamination it is recommended to start collecting blood after the removal of ascitic fluid and cease it once biliary anastomosis begins [34]. Another traditional contraindication for CS use is presence of malignancy due to a theoretical risk of reinfusion of salvaged blood contaminated with tumor cells. Data from retrospective analyses and small studies published so far in patients with hepatocellular carcinoma undergoing LT have showed that CS with leukocyte-depletion filters is effective and does not increase the risk of cancer reoccurrence. However, this needs to be confirmed in prospective, randomized trials [45].

\section{PHARMACOLOGICAL INTERVENTIONS}

\section{ANTIFIBRINOLYTIC DRUGS}

Fibrinolysis is an important process developing during anhepatic phase and progressing massively after reperfusion due to the alterations in haemostatic system explained above. Quite often it ceases spontaneously within the first hour after reperfusion, but may persist longer, especially in patients with poorly functioning grafts, leading to global microvascular oozing. Therefore, for many years antifibrinolytic 
drugs have been investigated extensively as a measure to reduce perioperative bleeding.

In 2011 a Cochrane review was published which nicely summarized the most important findings. However, as stated by the authors, due to lack of large, double-blinded, multicenter trials and high risk of bias, the quality of the evidence is considered relatively low [33]. It was demonstrated that aprotinin was the only antifibirinolytic agent to significantly reduce the requirements for RBCs, FFP, platelets and cryoprecipitate compared to a placebo [33]. Further trials comparing high with medium and low doses of aprotinin, as well as the mode of administration (bolus vs continuous infusion) have not revealed any differences in the perioperative blood loss and transfusion requirements. In 2007 aprotinin was withdrawn from European markets due to safety concerns after an interim analysis of data from a BART study comparing aprotinin with lysine analogues - tranexamic acid (TXA) and epsilon-aminocaproic acid (EACA) in patients undergoing cardiac surgery [46]. However, after review of the final results of the trial and all available data related to the safety of aprotinin, in 2012 the European Medicines Agency recommended that this suspension should be lifted, which was accepted by European Commission in 2013. The available data does not show that in LT recipients the use of aprotinin is associated with an increase in the rate of hepatic artery thrombosis, venous thromboembolism or mortality compared to a placebo $[33,47]$. Interestingly, a recently published retrospective analysis of patients routinely treated with aprotinin during LT compared to the period post aprotinin withdrawal, did not reveal any increase in the amount of RBCs, FFP, platelet concentrates and cryoprecipitate transfusion since the withdrawal of aprotinin [48].

A previously published meta-analysis demonstrated that TXA can also significantly reduce the amount of RBCs transfusion in LTs [49]. However, this observation was not confirmed by data from a Cochrane review [33]. Both of these papers did not reveal any negative impact of TXA on the risk of thromboembolic events or mortality [33, 49]. The EACA, which is widely used in the United States, had only one trial concerning LTs which did not show any benefit compared to a placebo [33]. According to the current guidelines, it is recommended to consider administration of antifibirinolytic drugs when fibrinolysis is either confirmed in viscoelastic tests (TEG/ROTEM) or is clinically evident from microvascular oozing, but not as a routine practice [34].

\section{RECOMBINANT ACTIVATED FACTOR VII}

Use of recombinant activated factor VII ( $\mathrm{rFVIla)}$ as a routine measure to reduce perioperative blood loss and transfu- sion requirements during LT have been investigated in three randomized controlled trials (RCT). Although they consistently showed correction of clotting abnormalities, rFVIla did not affect the requirements for RBCs, FFP or platelet concentrates compared to a placebo [33]. Despite concerns regarding an increased risk of arterial thrombosis associated with rFVlla use, the available data in LT recipients did not show any impact of this therapy on the risk of thromboembolic events, graft failure or other serious adverse effects [33]. Therefore, the ESA currently recommends against the prophylactic use of rFVIla during LT and only suggests its potential role as a "rescue therapy" to control massive haemorrhage [34].

\section{PROTHROMBIN COMPLEX CONCENTRATE}

Currently the first multicenter, randomized, doubleblinded trial comparing the routine use of prothrombin complex concentrate (PCC) with a placebo in patients with INR > 1.5 undergoing LT is pending [50]. In many centers, including our own, PCC is used "off-label" during LT as a rescue therapy during catastrophic bleeding, when coagulopathy is evident. Although the available data on safety in this population does not suggest an increased risk of thrombotic, thromboembolic and ischaemic events associated with PCC use, the data are scarce and need to be confirmed in large trials [51].

\section{FIBRINOGEN CONCENTRATE}

Introduction of viscoelastic tests quickly led to the conclusion that not only the quantitative deficits, but also the qualitative deficits of fibrinogen are a frequent feature in cirrhotic patients. A previously mentioned paper by Noval-Padillo et al. [42] demonstrated that up to $45 \%$ of LT recipients required fibrinogen concentrate administration to increase clot firmness, based on a ROTEM analysis. This treatment, when compared to historic controls, showed a marked decrease in the amount of RBCs, FFP and platelet concentrates transfusion, along with a significant increase in the rate of transfusion-free procedures [42]. Fibrinogen concentrate substitution was also found to restore MCF after in vitro hemodilution in blood from LT recipients, therefore suggesting its potential role in the treatment of dilutional coagulopathy [36]. In many centers cryoprecipitate is still used as the most abundant source of fibrinogen. It is now recommended that as fibrinogen concentrates contain standard doses of fibrinogen, as well as carrying lower risk of pathogen and immune mediated complications, they should be the preferred source of fibrinogen, in comparison to cryoprecipitate, for the treatment of the quantitative functional deficits of fibrinogen in bleeding patients, unless the former is unavailable [34]. 


\section{CONCLUSIONS}

Perioperative blood loss during LT is a complex endpoint which is affected not only by pre-existing alterations of the haemostatic system, but also by surgical and anaesthetic management. A multidisciplinary approach tailored for individual patients should be employed on case-to-case basis. Utilizing modern diagnostic and therapeutic options in order to decrease bleeding and the need for allogeneic blood product transfusion should lead to an improvement in global patient outcomes. Still more trials are required to assess the efficacy of novel management strategies.

\section{ACKNOWLEDGEMENTS}

1. The authors declare no financial disclosure.

2. The authors declare no conflict of interest.

\section{References}

1. Findlay JY, Long TR, Joyner MJ, Heimbach JK, Wass CT: Changes in transfusion practice over time in adult patients undergoing liver transplantation. J Cardiothorac Vasc Anesth 2013; 27:4145. doi: 10.1053/j.jvca.2012.06.004

2. Massicotte L, Denault AY, Beaulieu D et al.: Transfusion rate for 500 consecutive liver transplantations: experience of one liver transplantation center. Transplantation 2012; 93: 1276-1281. doi: 10.1097/TP.0b013e318250fc25.

3. Krzanicki D, Sugavanam A, Mallett S: Intraoperative hypercoagulability during liver transplantation as demonstrated by thromboelastography. Liver Transpl 2013; 19: 852-861. doi: 10.1002/lt.23668.

4. Brummel-Ziedins K, Mann KG: Molecular basis of blood coagulation. In: Hoffman R, Benz EJ Jr, Silberstein LE et al. (ed.): Hematology: basic principles and practice. Ed 6. Philadelphia. Sanders: Elsevier 2013: 1821-1841.

5. Marks PW: Hematologic manifestations of liver disease. Semin Hematol 2013; 50: 216-221. doi: 10.1053/j.seminhematol.2013.06.003.

6. Tripodi A, Mannucci PM: The coagulopathy of chronic liver disease. N Engl J Med 2011; 365: 147-156. doi: 10.1056/NEJMra1011170.

7. Weeder PD, Porte RJ, Lisman T: Hemostasis in liver disease: implications of new concepts for perioperative management. Transfus Med Rev 2014; 28: 107-113. doi: 10.1016/j. tmrv.2014.03.002.

8. Clevenger B, Mallett SV:Transfusion and coagulation management in liver transplantation. World J Gastroenterol 2014; 20 : 6146-6158. doi: 10.3748/wjg.v20.i20.6146.

9. Lisman T, Bakhtiari K, Adelmeijer J, Meijers JCM, Porte RJ, Stravitz $R T$ : Intact thrombin generation and decreased fibrinolytic capacity in patients with acute liver injury or acute liver failure. JThromb Haemost 2012; 10: 1312-1319. doi: 10.1111/j.15387836.2012.04770.x.

10. Lisman T, Porte R: Rebalanced hemostasis in patients with liver disease: evidence and clinical consequences. Blood 2010; 116 : 878-885. doi: 10.1182/blood-2010-02-261891.

11. Rana A, Petrowsky H, Hong JC et al.: Blood transfusion requirement during liver transplantation is an important risk factor for mortality. J Am Coll Surg 2013; 216: 902-907. doi: 10.1016/j. jamcollsurg.2012.12.047.

12. Boin IF, Leonardi MI, Luzo AC, Cardoso AR, Caruy CA, Leonardi LS: Intraoperative massive transfusion decreases survival after liver transplantation. Transplant Proc 2008; 40: 789-791. doi: 10.1016/j.transproceed.2008.02.058.

13. Massicotte L, Sassine MP, Lenis S, Seal RF, Roy A: Survival rate changes with transfusion of blood products during liver transplantation. Can J Anesth 2005; 52: 148-155.

14. de Boer MT, Christensen MC, Asmussen M et al.: The impact of intraoperative transfusion of platelets and red blood cells on survival after liver transplantation. Anesth Analg 2008; 106 32-44. doi: 10.1213/01.ane.0000289638.26666.ed.

15. Pereboom IT, de Boer MT, Haagsma EB, Hendriks HG, Lisman T, Porte RJ: Platelet transfusion during liver transplantation is associated with increased postoperative mortality due to acute lung injury. Anesth Analg 2009; 108: 1083-1091. doi: 10.1213/ ane.0b013e3181948a59.

16. Benson $A B$, Burton $J R$, Austin GL et al: Differential effects of plasma and red blood cell transfusions on acute lung injury and infection risk following liver transplantation. Liver Transpl 2011; 17: 149-158. doi: 10.1002/lt.22212.

17. Hendriks HG, van der Meer J, de Wolf JT et al.: Intraoperative blood transfusion requirement is the main determinant of early surgical re-intervention after orthotopic liver transplantation. Transpl Int 2005; 17: 673-679.

18. Araújo T, Cordeiro A, Proenca P, Perdigoto R, Martins A, Barroso E: Predictive variables affecting transfusion requirements in orthotopic liver transplantation. Transplant Proc 2010; 42: 1758-1759. doi: 10.1016/j.transproceed.2009.10.007.

19. Makroo RN, Walia RS, Aneja S, Bhatia A, Chowdhry M: Preoperative predictors of blood component transfusion in living donor liver transplantation. Asian JTransfus Sci 2013; 7: 140-146. doi: 10.4103/0973-6247.115586.

20. Mangus RS, Kinsella SB, Nobari MM et al.: Predictors of blood product use in orthotopic liver transplantation using the piggyback hepatectomy technique. Transplant Proc 2007; 39: 3207-3213.

21. Massicotte L, Beaulieu D, Roy JD et al.: MELD score and blood product requirements during liver transplantation: no link. Transplantation 2009; 87: 1689-1694. doi: 10.1097/ TP.0b013e3181a5e5f1.

22. De Santis GC, Brunetta DM, Nardo M et al.: Preoperative variables associated with transfusion requirements in orthotopic liver transplantation. Transfus Apher Sci 2014; 50: 99-105. doi: 10.1016/j.transci.2013.10.006.

23. Roullet $S$, Biais M, Millas E, Revel P, Quinart A, Sztark F: Risk factors for bleeding and transfusion during orthotopic liver transplantation. Ann Fr Anesth Reanim 2011; 30: 349-352. doi: 10.1016/j.annfar.2011.01.008.

24. Massicotte L, Beaulieu D, Thibeault L et al.: Coagulation defects do not predict blood product requirements during liver transplantation. Transplantation 2008; 85: 956-962. doi: 10.1097/ TP.0b013e318168fcd4.

25. McCluskey SA, Karkouti K, Wijeysundera DN et al.: Derivation of a risk index for the prediction of massive blood transfusion in liver transplantation. Liver Transpl 2006; 12: 1584-1593.

26. Steib A, Freys G, Lehmann C, Meyer C, Mahoudeau G: Intraoperative blood losses and transfusion requirements during adult liver transplantation remain difficult to predict. Can J Anaesth 2001; 48: 1075-1079.

27. Cheema SP, Hughes A, Webster NR, Bellamy MC: Cardiac function during orthotopic liver transplantation with venovenous bypass. Anaesthesia 1995; 50: 776-778.

28. Feltracco $P$, Brezzi $M$, Barbieri S et al.: Blood loss, predictors of bleeding, transfusion practice and strategies of blood cell salvaging during liver transplantation. World J Hepatol 2013; 5: 1-15. doi: 10.4254/wjh.v5.i1.1.

29. Vieira de Melo PS, Miranda LE et al: Orthotopic liver transplantation without venovenous bypass using the conventional and piggyback techniques. Transplant Proc. 2011; 43: 1327-1333. doi: 10.1016/j.transproceed.2011.03.061.

30. Gurusamy KS, Pamecha V, Davidson BR: Piggy-back graft for liver transplantation. Cochrane Database Syst Rev 2011; 1: CD008258. doi: 10.1002/14651858.CD008258.pub2.

31. Murthy TVSP: Transfusion support in liver transplantation. Indian J Anaesth 2007; 51: 13-19.

32. Palomo Sanchez JC, Jimenez C, Moreno Gonzalez E et al.: Effects of intraoperative blood transfusion on postoperative complications and survival after orthotopic liver transplantation. Hepatogastroenterology 1998; 45: 1026-1033.

33. Gurusamy KS, Pissanou T, Pikhart H, Vaughan J, Burroughs AK, Davidson BR: Methods to decrease blood loss and transfusion requirements for liver transplantation. Cochrane Database Syst Rev. 2011; (12): CD009052. doi: 10.1002/14651858.CD009052.pub2. 
34. Kozek-Langenecker SA, Afshari A, Albaladejo P et al.: Management of severe perioperative bleeding: guidelines from the European Society of Anaesthesiology. Eur J Anaesthesiol 2013; 30: 270-382. Review. Erratum in: Eur J Anaesthesiol 2014; 31: 247. doi: 10.1097/EJA.0b013e32835f4d5b.

35. Huntington JT, Royall NA, Schmidt CR: Minimizing blood loss during hepatectomy: a literature review. J Surg Oncol 2014; 109: 81-88. doi: 10.1002/jso.23455.

36. Nicińska B, Pluta J, Kosieradzki M et al.: The effects of in vitro hemodilution and fibrinogen concentrate substitution on thromboelastometry analysis in patients qualified for liver transplantation - preliminary results. Transplant Proc. 2014; 46: 2758-2761. doi: 10.1016/j.transproceed.2014.09.048.

37. Massicotte L, Lenis S, Thibeault L, Sassine MP, Seal RF, Roy A: Effect of low central venous pressure and phlebotomy on blood product transfusion requirements during liver transplantations. Liver Transpl 2006; 12: 117-123. Erratum in: Liver Transpl 2006; 12: 694

38. Hannaman MJ, Hevesi ZG: Anesthesia care for liver transplantation. Transplant Rev 2011; 25: 36-43. doi: 10.1016/j. trre.2010.10.004

39. Cywinski JB, Mascha E, You J et al.: Central venous pressure during the post-anhepatic phase is not associated with early postoperative outcomes following orthotopic liver transplantation. Minerva Anestesiol 2010; 76: 795-804.

40. Alamo JM, León A, Mellado P et al.: Is "intra-operating room" thromboelastometry useful in liver transplantation? A case-control study in 303 patients. Transplant Proc 2013; 45: 3637-3639. doi: 10.1016/j.transproceed.2013.11.008.

41. Wang SC, Shieh JF, Chang KY et al.: Thromboelastographyguided transfusion decreases intraoperative blood transfusion during orthotopic liver transplantation: randomized clinical trial. Transplant Proc 2010; 42: 2590-2593. doi: 10.1016/j. transproceed.2010.05.144.

42. Noval-Padillo JA, León-Justel A, Mellado-Miras Pet al.: Introduction of fibrinogen in the treatment of hemostatic disorders during orthotopic liver transplantation: implications in the use of allogenic blood. Transplant Proc 2010; 42: 2973-2974. doi: 10.1016/j.transproceed.2010.08.011.

43. Massicotte L, Thibeault L, Beaulieu D, Roy JD, Roy A: Evaluation of cell salvage autotransfusion utility during liver transplantation. HPB (Oxford). 2007; 9: 52-57. doi: 10.1080/ /13651820601090596.
44. Darwish A: Liver transplant in Jehovah's Witnesses patients. Curr Opin Organ Transplant. 2011; 16: 326-330. doi: 10.1097/ MOT.0b013e328346dd36.

45. Zhai B, Sun $X Y$ : Controversy over the use of intraoperative blood salvage autotransfusion during liver transplantation for hepatocellular carcinoma patients. World J Gastroenterol 2013; 19: 3371-3374. doi: 10.3748/wjg.v19.i22.3371.

46. Fergusson DA, Hébert PC, Mazer CD et al.: BART Investigators. A comparison of aprotinin and lysine analogues in highrisk cardiac surgery. N Engl J Med 2008; 358: 2319-2331. Erratum in: N Engl J Med 2010; 363: 1290. doi: 10.1056/ /NEJMoa0802395

47. Warnaar N, Mallett SV, Klinck JR et al.: Aprotinin and the risk of thrombotic complications after liver transplantation: a retrospective analysis of 1492 patients. Liver Transpl 2009; 15: 747-753. doi: 10.1002/lt.21768.

48. Schofield N, Sugavanam A, Thompson K, Mallett SV: No increase in blood transfusions during liver transplantation since the withdrawal of aprotinin. Liver Transpl 2014; 20: 584-590. doi: 10.1002/lt.23839.

49. Molenaar IQ, Warnaar N, Groen H, Tenvergert EM, Slooff MJ, Porte RJ: Efficacy and safety of antifibrinolytic drugs in liver transplantation: a systematic review and meta-analysis. Am J Transplant 2007; 7: 185-194.

50. Arshad $F$, Ickx B, van Beem RT et al.: Prothrombin complex concentrate in the reduction of blood loss during orthotopic liver transplantation: PROTON-trial. BMC Surg 2013; 13: 22. doi: 10.1186/1471-2482-13-22.

51. Kirchner C, Dirkmann D, Treckmann JW et al.: Coagulation management with factor concentrates in liver transplantation: a single-center experience. Transfusion 2014; 54: 2760-2768. doi: $10.1111 /$ trf. 12707

\section{Corresponding author:}

Małgorzata H. Starczewska, MD

$2^{\text {nd }}$ Department of Anaesthesia and Intensive Care

Central Teaching Hospital, Medical University of Warsaw

ul. Banacha 1a, 02-097 Warsaw, Poland

e-mail: starczewska@gmail.com

Received: 17.02.2015 r.

Accepted: $1.04 .2015 \mathrm{r}$. 